\title{
Laparoscopic closure of perforated gastro-duodenal ulcer: 15 years' experience in our centre
}

\author{
Michal Žáček, Juraj Ván̆a, Boris Babiš \\ Department of Surgery, The Faculty Hospital, Žilina, Slovak Republic
}

Videosurgery Miniinv 2014; 9 (4): 578-585 DOI: $10.5114 /$ wiitm.2014.45888

\begin{abstract}
Introduction: The objective of the study is to share the results and development findings on the laparoscopic closure technique applied in our centre during a 15-year period (1998-2012).

Aim: To compare statistically the standard parameters (hospitalization, duration of operation) versus conventional surgery, and at the same time we compared mainly morbidity and mortality.

Material and methods: During the period under review we operated on a total of 259 patients, 115 (44.4\%) of them laparoscopically, and 144 (55.6\%) of them conventionally. The sample was divided into two groups: patients with ASA physical status classification system 1-3, and patients with ASA 4-5.

Results: The results favour laparoscopy within the group with ASA 1-3 in terms of several parameters, namely: duration of hospitalization - 7.7 days in the case of laparoscopic intervention, vs. 10.6 days for conventional surgery ( $p<0.05)$; and duration of operation $-61 \mathrm{~min} v$ s. $85.1 \mathrm{~min}$ respectively $(p<0.05)$. Total morbidity was $27.5 \%$ in the case of patients with conventional surgery, vs. $10.9 \%$ with laparoscopic intervention $(p<0.05)$. The sample of patients with ASA 4-5 suffered a high mortality of $82.7 \%$.

Conclusions: Laparoscopic closure of perforated ulcer is a safe therapeutic method, as confirmed by the results of many other studies around the world, which in many aspects favour the laparoscopic technique.
\end{abstract}

Key words: peptic ulcer, gastroduodenal perforation, laparoscopy, laparoscopic suture, laparoscopic closure.

\section{Introduction}

Johan Mikulicz-Radecki (1850-1905), often referred to as the first surgeon who closed a perforated peptic ulcer by simple closure, said: 'Every doctor, faced with a perforated duodenal ulcer of the stomach or intestine, must consider opening the abdomen, sewing up the hole, and averting a possible inflammation by careful cleansing of the abdominal cavity' [1].

Perforation occurs in approximately $2-10 \%$ of patients with ulcer disease [2]. It comprises approximately $5 \%$ of all abdominal emergencies, and perforation incidence is $7-10$ per 100000 capita [3, 4].

Patients with perforated ulcer are predominantly men aged 40 to 60 years [4]. They could have ulcer disease anamnesis (29\%), or consumption of NSAID (20\%). Around $5-10 \%$ of patients arrive at the hospital in a condition of shock [5]. On the other hand, one of the rarest causes of stomach perforation is incarceration of hiatus hernia $(<0.01 \%)[6]$.

X-ray examination performed in a standing position will in $80-85 \%$ of cases prove the presence of free air under the diaphragm, and the subsequent radiological techniques confirm the diagnosis in $80-$ $90 \%$ of cases [7].

It clinically manifests as a sudden, from the very beginning unusually intense, shocking pain, with abdominal guarding clinical symptoms.

\section{Address for correspondence}

Michal Žáček MD, Department of Surgery, The Faculty Hospital, V. Spanyola 43, 04301 Žilina, Slovak Republic, phone: +42 1908537194,

e-mail: michalzacek@yahoo.com 
Clinically we distinguish three stages [8]:

1. Chemical peritonitis - acidic content sterilises the content of stomach and duodenum, which freely flows to the peritoneum, and causes the chemical peritonitis.

2. Transitory stage - after 6-12 h there occurs an arbitrary improvement - pain relief, caused by dilution of gastro-duodenal liquid with peritoneal exudate.

3. Intra-abdominal infection emerges after 12 to $24 \mathrm{~h}$. Post-surgery mortality in the case of perforated ulcer still remains high, around $6-10 \%$ [9]. Boey or Irvin score systems (0-3 scale, Table I) relate mortality risk to such factors as:

1. Condition of shock before surgery -1 point + ,

2. Dominant associated disease -1 point + ,

3. Postponement of surgical intervention by more than $24 \mathrm{~h}$ from the beginning of the disease 1 point + .

\section{Laparoscopic treatment of perforated ulcer}

The first laparoscopic suture closure was carried out by Nathanson et al. in 1990 [10]. At the same time Mouret carried out the first laparoscopic omentoplastic surgery using a fibrin sealant [11]. From available references on the data collected through questionnaires, in 2004 in Slovak Republic only $24.2 \%$ of relevant surgery departments applied laparoscopic suture closure of perforated gastro-duodenal ulcer [12], whereas in the Czech Republic the same figure in 2002 was already 56\% [13].

In the beginning the laparoscopic approach is indicated in each suspected case of perforated ulcer, but it also has counter indications, such as a highrisk patient with ASA 5, and ileus.

\section{Position of a patient and position of the operating team}

In this intervention the patient lies on his/her back, with the left upper extremity adducted. The operating surgeon is on the left side of the patient, and the surgeon's assistant is on the left side of the surgeon. The operating surgeon may eventually be between the patient's legs, and the surgeon's assistant in this case remains on the left side of the patient [14]. The laparoscopic tower is on the right side of the patient, next to his/her chest, or head. This setup is good for preparation in the epigastric region. The position of the patient, and/or his/her rotation during the intervention should be as follows: During the preparation carried in the epigastric region, the patient should be in an anti-Trendelenburg position at $20-30^{\circ}$ [15], which provides a better view of the operating field, since the viscera fall down. In case of cleansing the patient can be leaned in different directions, depending on the necessity to visually inspect individual abdominal quadrants.

\section{Position of trocars}

We usually insert the optical $10 \mathrm{~mm}$ port into the umbilicus. The working $5 \mathrm{~mm}$ port for the operating surgeon's left hand is located in the anterior axillary line at the level of the umbilicus, for the atraumatic grasper. Another $5 \mathrm{~mm}$ working port for the operating surgeon's right hand is located in the medio-clavicular line above the level of the umbilicus for the suture holder, and the suction and irrigation device (Figure 1).

In the case of obese patients, the position of ports can be adjusted and moved closer to the operating field. In the case of a bad view of the local situation, a fourth port can be located in the epigastrium, for a retractor for the liver and viscera.

\section{Surgical procedure during suture closure of a perforated ulcer}

The next step after the introduction of an optical port and confirmation of the diagnosis is the introduction of working ports as described above. First we take a sample of exudate for bacteriological tests, and then we carry out the inspection of the abdominal cavity, in order to localise precisely the perforation spot, and the extent of peritonitis. Quite often the gall bladder and the liver adhere with fibrin accretions in the vicinity of the ulcer, which is most frequently located on the frontal side of the first part of the duodenum. The follow-up step is the cleansing of the abdominal cavity, where irrigation with a warm physiological solution is followed by

Table I. Boey score system with respect to morbidity and mortality

\begin{tabular}{|ccc|}
\hline Boey & Morbidity [\%] & Mortality [\%] \\
\hline 0 & 17.4 & 1.5 \\
\hline 1 & 30.1 & 14.4 \\
\hline 2 & 42.1 & 32.1 \\
\hline 3 & & 100 \\
\hline
\end{tabular}




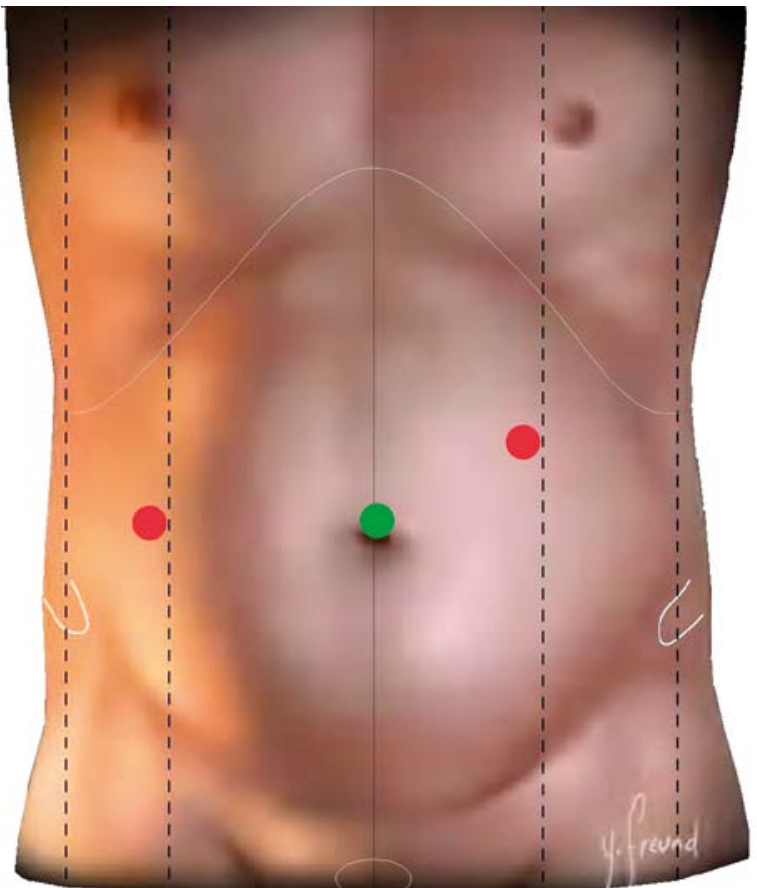

Figure 1. Position of ports during suturing

evacuation of the exudate and removal of fibrin accretions to the maximum possible extent. In terms of methodology we start at the right upper quadrant, proceeding to the left upper one, then we continue to the left lower quadrant, and we end in the right lower one. We need to be especially diligent in the area of the Douglas cavity, and in the space between intestinal loops.

\section{Perforation suture closure technique}

After the suture closure of the ulcer, there is no need for biopsy of the duodenal ulcer, but on the other hand, in the case of gastric ulcer, it is recommended to take a biopsy of the ulcer margin. Suture is carried out by a slowly absorbable or non-absorbable material applied with atraumatic needle $2 / 0$ or $3 / 0$. Usually two or three transverse sutures are applied (Photos 1 and 2). After the perforation is suture-closed, it is possible (using a part of the large omentum) to cover the suture closure, and fix it to the upper suture. Some surgeons apply for omentoplastic intervention a fibrin sealant. In the case of a chronic callous ulcer, it is problematic to sew together ulcer margins, and we can therefore apply a thicker thread, 1/0, in order to avoid it cutting through the tissue in a fibrous environment.



Photo 1. Perforation suture A

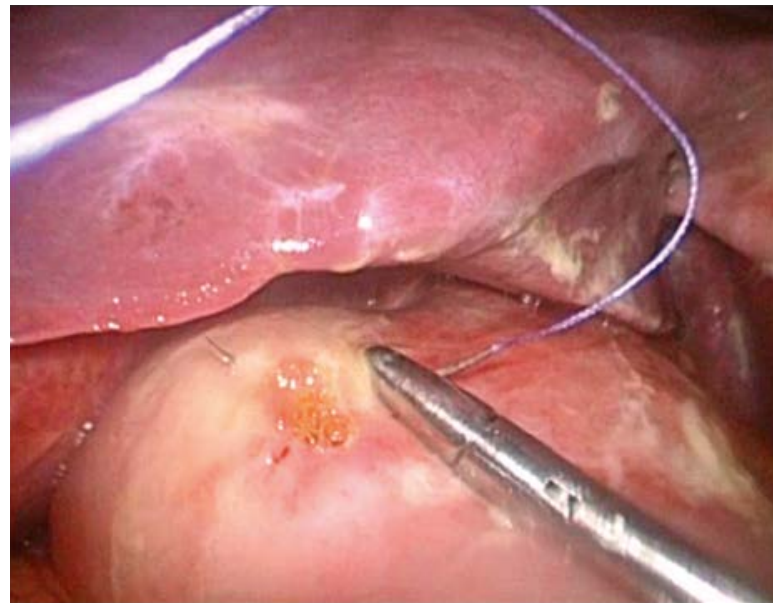

Photo 2. Perforation suture B

In the case of larger ulcers it is possible to sew-in within the defect, with several stitches, the free end of the omentum, in order to close the defect. It is the very size of the defect that causes the conversion [16].

We can check the tightness of the suture closure with a patient in the Trendelenburg position, following the application of physiological solution and blowing air into the stomach, which should not cause air leakage into the free abdominal cavity.

Flushing of the abdominal cavity is performed until the clear liquid comes out, and then we end the operation by applying drain tubes [15], which we place in the following order. One goes into the subhepatic space, which monitors the area of suture, which goes into the incision used as a port in the right mesogastrium. The remaining two drain tubes are inserted from the left, through incisions used as ports into the left subphrenic space and to 
the Douglas cavity (in the case of 3-port intervention we introduce into the Douglas cavity a drain tube through an incision on the right side). After the operation we keep in place the nasogastric tube until the peristaltic restart onset. Provided the clamping test was successful, the tube can be extracted. In complicated ulcer-suturing cases, or protracted onset of passage, we can carry out examination using a water contrast agent (UroDiagramin). The gastro-fibroscopic examination is planned following 4 to 6 weeks after the operation.

\section{Material and methods}

During the 15-year period from 1998 until 2012, we carried out in our centre altogether 259 operations of perforated gastro-duodenal ulcer. We did operations laparoscopically in the case of 115 patients $(44.4 \%)$, and conventionally in the case of 144 patients (55.6\%). The best method for comparing the two groups is the comparison of morbidity and mortality. For group homogeneity reasons we compared separately patients with ASA 4-5, whom we put aside from the compared groups. These patients suffer high mortality and mortality, with a high number of cases with high entry cardio-pulmonary premorbidity as shock-affected patients, who were in most cases classified as patients with contraindicated laparoscopic revision, which would substantially change the outcome for the group of patients operated conventionally (Table II). There were altogether 29 patients with ASA 4-5 (11.1\%).

The number of conventionally operated patients with ASA 1 during the period from 1998 until 2012

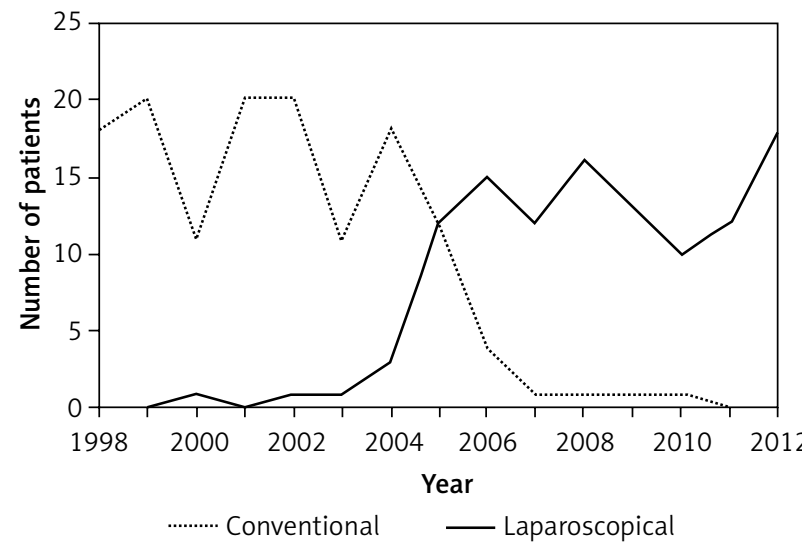

Figure 2. Ratio of laparoscopic intervention versus conventional surgical therapy of the perforated ulcer in our department
Table II. Distribution of the group of patients with respect to ASA

\begin{tabular}{|lccc|}
\hline Group & ASA 1-3 & ASA 4-5 & Together \\
\hline Conventional & 121 & 24 & 145 \\
\hline Laparoscopic & 110 & 5 & 115 \\
\hline
\end{tabular}

was 120 , and for laparoscopically operated patients it was 110 . The homogeneity of the group ensures a smooth transition to laparoscopy (Figure 2), when the surgeons in our centre (following sufficient training) started to choose laparoscopic inspection as the preferred choice.

\section{Group of conventionally operated patients with ASA 1-3}

The majority of patients from the group of 120 conventionally operated patients were operated on between 1998 and 2004-2005. In those years in the course of conventional surgical therapy of perforated ulcer the most frequent intervention was excision of the ulcer followed by pyloroplasty (93 patients: $77.5 \%)$, as well as associated truncal subdiaphragmatic vagotomy (60 patients, 50\%). Not in all cases of pyloroplasty did we also do vagotomy, and vice versa (Table III). In a smaller number of cases we did partial resection of the stomach and gastroenterostomy (altogether 6 patients, 5\%). Vagotomy had a complicated course in 2 patients with spleen lesion, i.e. in $3.3 \%$ of all vagotomies, with the subsequent need to carry out splenectomy. The average duration of hospitalisation was 10.6 days, and the average duration of surgical intervention was $85 \mathrm{~min}$.

The total number of patients with complications within the group of conventionally operated patients, with ASA 1-3, was 33 (27.5\%). Among them 4 patients died (3.33\%). Within this group (in several cases there were multiple complications per single patient) the most frequent complication was wound infection, and more specifically in the case of 14 (11.7\%) pa-

Table III. Percentage of individual types of interventions in conventionally operated patients with ASA $1-3(n=120)$

\begin{tabular}{|lcc|}
\hline Intervention & Patients & Percentage \\
\hline Pyloroplasty & 93 & 77.5 \\
\hline Vagotomy & 60 & 50 \\
\hline BII + GEA & 6 & 5 \\
\hline
\end{tabular}


tients. Extra-abdominal complications (bronchopneumonia or cardiac decompensation) occurred in 11 (9.2\%) patients, of whom 2 patients died. We re-operated 11 (9.2\%) patients, of whom 2 died. The reason for re-operation was $2 \times$ ileus, $3 \times$ intra-abdominal abscess, $2 \times$ disruptions of wound, and $3 \times$ leakages. Other complications occurred in 2 patients (bleeding).

We identified leakage in 3 patients (of whom 1 died), whereas in all cases re-operation was required, which comprises $2.5 \%$ of the total number.

The average duration of hospitalisation of a patient with complications in conventionally operated cases with ASA 1-3 within this group was 16.8 days vs. 8.2 days on average for patients without complications.

\section{Group of laparoscopically operated patients with ASA 1-3}

The majority of patients from the group of 110 laparoscopically operated patients were operated on between 2004-2005 and 2012 (Figure 2). In the case of laparoscopically operated patients with perforated ulcer, 81 patients were suture closed (73.6\%), sutured ulcer with subsequent omentoplasty was carried out in 17 patients (15.5\%), and the remaining 12 patients (10.9\%) were treated only with flushing of the abdominal cavity, and a targeted drain tube without suture due to failure to find the perforation (with already healing ulcer - application of methylene blue via nasogastric tube) (Table IV).

The average duration of hospitalisation was 7.7 days, and the average duration of intervention was $61 \mathrm{~min}$.

Table IV. Percentage of individual types of interventions in laparoscopically operated patients with ASA $1-3(n=110)$

\begin{tabular}{|lcc|}
\hline Intervention & Patients & Percentage \\
\hline Simple closure & 81 & 73.6 \\
\hline Suture + omentoplasty & 17 & 15.5 \\
\hline Targeted drainage & 12 & 10.9 \\
\hline
\end{tabular}

Table V. Mortality comparison for patients with ASA 4-5

\begin{tabular}{|lccc|}
\hline Group & ASA 4-5 & Exitus & Exitus [\%] \\
\hline Conventional & 24 & 20 & 83.30 \\
\hline Laparoscopic & 5 & 4 & 80 \\
\hline
\end{tabular}

The total number of patients with complications within the group of laparoscopically operated patients with ASA 1-3 was 12 (10.9\%). Among them 1 patient died $(0.9 \%)$ (heart failure, pulmonary embolism). We did not record any wound infection within the group of 12 patients with complications. Bronchopneumonia or cardiac decompensation occurred in 4 patients (3.6\%). Operative re-intervention was required in 4 (3.6\%) patients. The reason for surgical revision was in 2 cases an intra-abdominal abscess, and in 2 others leakage. Of the remaining 4 patients (33\%), conservative therapy was applied in 2 patients with leakage, and in 2 patients with an intra-abdominal abscess.

Altogether, leakage occurred in 4 patients, which comprises $3.6 \%$ of the total group of patients, but only in 2 cases, i.e. $1.8 \%$, did it require re-operation.

Conversion occurred in $2(1.8 \%)$ patients. The reasons for conversions were polyps in 1 case, and the size of the ulcer in another single case.

The average duration of hospitalisation in patients with complications among laparoscopically operated patients with ASA 1-3 within this group was 15.7 days vs. an average of 6.7 days in patients without complications.

\section{Group of operated patients with ASA 4-5}

From 1998 until 2012, 29 (11.1\% of all) patients with ASA 4-5 underwent operations. They suffered high morbidity and mortality. This was the case due to cardiac reasons, or the associated status of shock during the protracted course of peritonitis (within our group the postponement of hospitalisation by patients themselves) (Table V). The mortality within this group was $82.7 \%$, i.e. death occurred in 24 patients. In the case of the majority of patients we could not carry out laparoscopy due to the anaesthesiology risk of capnoperitoneum (the main reason behind the exclusion of this group). The laparoscopy in the case of the remaining 5 of these patients did not influence the haemodynamics during the course of the operation.

\section{Statistical analysis}

For the statistical assessment of parametric quantitative values we used Student's $t$ test (bilateral distribution with uneven dispersions), and for the assessment of qualitative values we used the $\chi^{2}$ test with a $2 \times 2$ contingency table, with the statistical significance limit level being $p<0.05$. The minimal number of patients within a single contingency table 
should be at least 5 , in order to maintain the test accuracy. Provided the number of patients was below 5 , we did not assess the said qualitative parameter from the viewpoint of statistical significance.

\section{Results}

In addition to the basic parameters such as comparison of hospitalisation duration, and operating time (Table VI), we focused mainly on the comparison of morbidity and mortality, which are the main distinctions in comparing the two methods, i.e. laparoscopic and conventional operation approaches (Table VII). As mentioned above, for group homogeneity reasons, we only compared patients with ASA 1-3 (88.9\% of all patients).

These statistical comparison results show a significant difference in the hospitalisation duration in favour of laparoscopy, on average 7.7 days vs. 10.6 days for conventionally operated patients. Similarly the operation intensity in terms of operation duration confirmed a statistically significant difference in favour of laparoscopy, namely $61 \mathrm{~min}$ vs. $85 \mathrm{~min}$ for conventionally operated patients. The average age was comparable.

We found a statistically significant difference in the number of complications in favour of laparoscopy, $10.9 \%$ vs. $27.5 \%$ for conventional operations. In the case of laparotomy we did not record even a single wound infection complication compared to the conventional group with the occurrence of $11.7 \%$.

The differences in favour of laparoscopy, 3.6\% vs. $9.2 \%$ in the case of postoperative occurrence of extra-abdominal complications, such as bronchopneumonia and cardiac complications, were statistically insignificant. The differences are apparent, but for statistical confirmation they would normally require a larger group of patients. Similarly to the complications with the follow-up necessity of postoperative re-intervention, laparoscopy seems to be the preferred choice, with $3.6 \%$ vs. $9.2 \%$ in the case of conventionally operated patients. Even in this case, for the confirmation of a significant difference we would require a larger group.

With very low numbers of patients in leakage and exitus groups, we were unable to compare the results, although the results were similar.

\section{Discussion}

The best parameters for comparison of the two operating techniques are mortality and morbidity. Perforated ulcer is still associated with a high rate of morbidity and mortality. Several studies have been published, although only 3 of them are prospectively randomized [17-19], with a high (degree of evidence). The comparison of results in the

Table VI. Statistical comparison of individual parameters in laparoscopic and conventional groups (operating time, hospitalisation duration, average age)

\begin{tabular}{|lcccc|}
\hline Parameter & Conventional & Laparoscopic & Value of $p$ & Statistical significance \\
\hline Operating time [min] & 85.1 & 61 & 0.0000000004 & $p<0.05$ \\
\hline Hospitalisation [days] & 10.6 & 7.7 & 0.00013 & $p<0.05$ \\
\hline Average age [years] & 48.6 & 47.2 & 0.52 & \\
\hline
\end{tabular}

Table VII. Statistical comparison of individual parameters in laparoscopic and conventional groups (morbidity and mortality)

\begin{tabular}{|c|c|c|c|c|}
\hline Parameter & $\begin{array}{l}\text { Conventional } \\
\quad(n=120)\end{array}$ & $\begin{array}{l}\text { Laparoscopic } \\
\quad(n=110)\end{array}$ & Value of $p$ & $\begin{array}{c}\text { Statistical } \\
\text { significance }\end{array}$ \\
\hline Complications: & $33(27.5 \%)$ & $12(10.9 \%)$ & 0.0015 & $p<0.05$ \\
\hline Exitus & $4(3.3 \%)$ & $1(0.9 \%)$ & - & \\
\hline Wound infection & $14(11.7 \%)$ & 0 & 0 & $p<0.05$ \\
\hline Bronchopneumonia & $11(9.2 \%)$ & $4(3.6 \%)$ & 0.09 & \\
\hline Re-intervention & $11(9.2 \%)$ & $4(3.6 \%)$ & 0.09 & \\
\hline LEAK & $3(2.5 \%)$ & $4(3.6 \%)$ & - & \\
\hline
\end{tabular}


aforementioned studies shows significant differences in morbidity (22\% in laparoscopy group vs. $36 \%$ in conventional group), and mortality ( $2.5 \%$ vs. $5.8 \%)$.

In these studies postoperative leakage occurred more frequently in the laparoscopic group (3\%), versus the conventional group, with only $1.1 \%$ of operated patients with leakage [17-19]. However, it is necessary to realise that laparoscopic suture closure of the perforated ulcer is a relatively complicated operation, and it requires manual skills in mini-invasive surgery.

The reason for conversion, $12.4 \%$ on average, is in most cases the size of the perforation [16]. Additional reasons were inability to visualise the perforation, location of perforation, and bleeding.

In one of the most recent studies, there were not found any significant differences in terms of post-operative leakage from the suture closure, when the surgeons applied a single suture without omentoplasty, or with it [20].

Alternative options are the application of fibrin glue, which is limited by the size of the perforation, or the application of biodegradable lactide-glycolide-caprolactone patches, which are still at the stage of development.

During the recent period, we can observe a gradual cessation of the final surgical intervention (resection, pyloroplasty, vagotomy) with low re-occurrence of the ulcer disease following the eradication of Helicobacter pylori (double combination of antibiotics and proton-pump inhibitor), and elimination of NSAID usage [21].

\section{Conclusions}

Laparoscopic suture closure of the perforated gastro-duodenal ulcer performed in a centre with sufficient history in laparoscopic surgery is a safe method, which offers better results compared to conventional therapy. The potential advantages of the mini-invasive approach are as follows: better view of the trans-operative situation, limited surgical trauma, smaller operating wound, limited intestinal manipulation and earlier recovery to baseline activities [22]. These advantages contribute to the lower occurrence of complications, especially such as wound complications, and the method is associated with reduced postoperative pain, and the possibility of faster mobilisation. In economic terms, laparoscopy provides a shorter hospitalisation time, and last but not least, a better cosmetic outcome. Even the conclusion of the EAES consensus as of 2006 reads that in the case of suspected perforated ulcer it is recommended to apply a mini-invasive approach as a safe therapeutic method [23]. This has also been confirmed by available prospective randomised studies, and in our centre the laparoscopic suture closure became the option of choice.

\section{References}

1. Schein M. Perforated peptic ulcer. Schein's common sense emergency. Abdominal Surgery 2005; 3: 143-50.

2. Druart ML, Hee RV, Etienne J, et al. Laparoscopic repair of perforated duodenal ulcer: a prospective multicenter clinical trial. Surg Endosc 1997; 11: 1017-20.

3. Ramakrishnan K, Salinas RC. Peptic ulcer disease. Am Fam Physician 2007; 76: 1005-12.

4. Lunevicius R, Morkevicius M. Management strategies, early results, benefits and risk factors of laparoscopic repair of perforated peptic ulcer. World J Surg 2005; 29: 1299-310.

5. Lagoo S, McMahon RL, Kakihara M, et al. The sixth decision regarding perforated duodenal ulcer. JSLS 2002; 6: 359-68.

6. Váňa J. Upside down stomach and its complications [Slovakian]. Slov Chir 2012; 9: 115-6.

7. Lau WY. Perforated peptic ulcer: open versus laparoscopic repair. Asian J Surg 2002; 25: 267-9.

8. Bertleff MJ, Lange JF. Perforated peptic ulcer disease: a review of history and treatment. Dig Surg 2010; 27: 161-9.

9. Imhof M, Epstein S, Ohmann C, Röher HD. Duration of survival after peptic ulcer perforation. World J Surg 2008; 32: 408-12.

10. Nathanson LK, Easter DW, Cuschieri A. Laparoscopic repair peritoneal toilet of perforated duodenal ulcer. Surg Endosc 1990; 4: 232-3.

11. Mouret P, François Y, Vignal J, et al. Laparoscopic treatment of perforated peptic ulcer. Br J Surg 1990; 77: 1006.

12. Johanes R, Holéczy P, Hamžík J. Past, present, and future of the slovak laparoscopy [Slovakian]. Slov Chir 2006; 3: 9-15.

13. Duda M, Gryga A, Czudek S, Skalický P. Twenty years of minimally invasive surgery in the Czech Republic. Videosurgery Miniinv 2011; 6: 42-7.

14. Czubek W, Januszkiewicz M, Wasilczuk M. Laparoscopic repair of perforated peptic ulcer - our own experience. Videosurgery Miniinv 2007; 2: 103-7.

15. Wróblewski T, Krawczyk M. Laparoscopic surgery in the treatment of acute abdomen [Polish]. Post N Med 2006; 19: 48-52.

16. Bertleff MJ, Lange JF. Laparoscopic correction of perforated peptic ulcer: first choice? A review of literature. Surg Endosc 2010; 24: 1231-9.

17. Siu WT, Leong HT, Law BK, et al. Laparoscopic repair for perforated peptic ulcer, a randomized controlled trial. Ann Surg 2002; 235: 313-9.

18. Lau WY, Leung KL, Kwong KH, et al. A randomized study comparing laparoscopic versus open repair of perforated peptic ulcer using suture or sutureless technique. Ann Surg 1996; 224: 131-8. 
19. Bertleff MJ, Halm JA, Bemelman WA, et al. Randomized clinical trial of laparoscopic versus open repair of the perforated peptic ulcer: the LAMA trial. World I Surg 2009; 33: 1368-73.

20. Lo HC, Wu SC, Huang HC, et al. Laparoscopic simple closure alone is adequate for low risk patients with perforated peptic ulcer. World J Surg 2011; 35: 1873-8.

21. Strzałka M, Bobrzyński A. Laparoscopy in the treatment of acute abdominal diseases. Videosurgery Miniinv 2008; 3: 1-9.

22. Šoltés M, Radoňak J. Current management of perforated peptic ulcer - what is the evidence? [Slovakian]. Slov Chir 2012; 9 146-50.

23. Neugebauer EAM, Sauerland S, Fingerhut A, et al. EAES Guidlines for endoscopic surgery, twelve years evidence-based surgery in Europe. Springer Berlin Heidelberg 2006; 338-9.

Received: 10.08.2014, accepted: 10.09.2014. 